\title{
Epipolar Resampling of Pushbroom Satellite Images Using Piecewise Linear Implementation of Pseudo Epipolar Line
}

\author{
Dong-Min Woo ${ }^{1}$ and Thanh-Danh Pham ${ }^{2}$ \\ ${ }^{1,2}$ Department of Electronics Engineering, Myongji University \\ Yongin, Gyeonggido, Korea 17058 \\ 1'dmwoo2000@gmail.com,2ptdanh392@gmail.com
}

\begin{abstract}
This paper presents a practical and efficient epipolar resampling method for pushbroom satellite images. We proposed a new resampling scheme based on PEL (Pseudo Epipolar Line). The main concept of PEL is derived from the assumption that the projection line from the satellite image sensor is a straight line. In this context, the epipolar line can be assumed as PEL, which can be directly evaluated directly from RPC (Rational Polynomial Coefficient) model. The pushbroom satellite image sensor uses a linear array scanner for image capture. Since the resulting images are formed by collecting the one-dimensional line images with the sensor movement, the line assumption is best approximated in the vicinity of the area forming the one dimensional line image. In this regard, we devise a piecewise linear implementation approach of the PEL epipolar resampling method to best approximate the epipolarity model of the linear pushbroom image. In this new approach, whole satellite images are divided into small images (pieces), and epipolar resampling is performed on each individual piece using the PEL. This approach is very accurate because the epipolar lines in each small image are evaluated by conjugate points within the terrain corresponding to the small image. Experimental results show that highly accurate resampling was achieved when all the whole images are divided into multiple small pieces. Our study confirmed that the suggested method can be efficiently utilized in epipolar image generation.
\end{abstract}

Keywords: Epipolar resampling, Pseudo epipolar line, Satellite image, Rational polynomial coefficient, Piecewise linear

\section{Introduction}

The principle of epipolar geometry was used to transform the original pair into a new image in an epipolar plane. Epipolar geometry is the intrinsic projective geometry between two views. It is independent of scene structure and depends on the camera perspective [1].

The IKONOS satellite consists of an optical system that has a pushbroom sensor [2]. It depends on epipolar geometry to acquire images of the earth's surface [3]. The IKONOS satellite collects images of the same area with the satellite in two other positions on the same orbit. From these two images, the epipolar geometry is applied for stereo reconstruction. The epipolar line formed by pushbroom epipolarity is represented not by a straight line but by a hyperbolic curve [4][5].

Ono [6] applied a well-established 2D affine orientation model to the epipolar resampling of satellite imagery. Using a 2D Affine Orientation Model, he developed an improved the epipolar resampling method that generated epipolar images without the knowledge of the Digital Terrain Model (DTM) or rigorous geometric parameters for reduction of vertical parallaxes. The resampling process is carried out along the epipolar

Received (December 11, 2017), Review Result (March 8, 2018), Accepted (March 12, 2018) 
line because the vertical parallax is due to a transformation error in the scanning direction. It was reported that the RMS value of vertical parallaxes between a pair of stereo epipolar images was less than half a pixel.

Morgan et al., [7][8] proposed an approach for epipolar resampling using parallel projection. In this approach, they used Exterior Orientation Parameters (EOP) and Interior Orientation Parameters (IOP) to resample satellite images. To obtain the parameters associated with the rigorous model, they performed an indirect estimation. This parallel projection method is attractive because it involves few parameters, which can be determined using a limited number of ground control points.

Wang et al., [9] described a practical epipolarity model for high-resolution linear pushbroom satellite images acquired in either an along-track model or a cross-track model based on the Projection Reference Plane (PRP) in object space, and this model was used for epipolar resampling. The pixel-to-pixel relationship between the original image and the generated epipolar image was established directly by the geometric sensor model using the Rational Function Model (RFM) to determine the corresponding image point. The results indicated that the model can serve as another strategy to determine the approximate line direction of the epipolar curve locus on the PRP. However, when the pair of conjugate image points used here happens to intersect an object point on or quite near the PRP, unstable or incorrect results are derived.

Woo [10] provided a procedure for the conjugate epipolar curve pair determination and Pseudo Epipolar Line (PEL) estimation of pushbroom images directly from a Rational Polynomial Coefficient (RPC) model. The proposed method assumes that the conjugate epipolar curve pairs exist and then epipolar image resampling is established by reassigning the generated conjugate epipolar curve pair points to satisfy the epipolar resampling condition. The epipolar resampling method using RPCs with the local epipolar curve can be approximated by a PEL under the assumption that the projection line from the satellite image sensor is linear. The concept of PEL was later applied to stereo matching procedure [11].

A piecewise epipolar curve generation method [12][13] was proposed based on a straight line approximation and higher-order polynomial fitting to attain sub-pixel accuracy. However, the epipolar curve was created by the height range, and as the height range increased, the deviation increased up to more than five pixels. Furthermore, epipolar lines could be determined by conjugate points far from the approximated epipolar line. This disadvantage leads to unstable and inaccurate epipolar line approximation.

This paper proposes a piecewise approach for epipolar resampling using piecewise linear rational functions. Since epipolar images are formed by collecting the onedimensional line images with the sensor movement, the line assumption is best approximated in the vicinity of the area forming one dimensional line images. In this context, we divide the whole image into small pieces and then find epipolar lines on each piece using conjugate points within the corresponding area.

\section{Epipolarity Formulation}

\subsection{Epipolar Geometry}

The epipolar geometry between two views is essentially the geometry of the intersection of the image planes with the pencil of planes with the baseline as the axis (the baseline is the line joining the camera centers). This geometry is usually motivated by considering the search for corresponding points in stereo matching, and here we will start from that objective.

Suppose a point $\mathrm{X}$ in the space is imaged in two views, at $\mathrm{X}_{\mathrm{L}}$ in the first, and at $\mathrm{X}_{\mathrm{R}}$ in the second. $\mathrm{O}_{\mathrm{L}}$ and $\mathrm{O}_{\mathrm{R}}$ represent the centers of projection of the two cameras. Points $\mathrm{X}_{\mathrm{L}}$ 
and $X_{R}$ are the projections of point $X$ onto the image planes that is denoted as $\pi$. Since the centers of projection of the cameras are distinct, it projects onto a point in the other image plane. The two image points are denoted by $\mathrm{e}_{\mathrm{L}}$ and $\mathrm{e}_{\mathrm{R}}$ and are called epipolar points. The epipolar line is the intersection of an epipolar plane with the image plane. All epipolar lines intersect at the epipole. An epipolar plane intersects the left and right image planes in epipolar lines, and defines the correspondence between the lines. Examples of epipolar geometry are given in Figure 1.

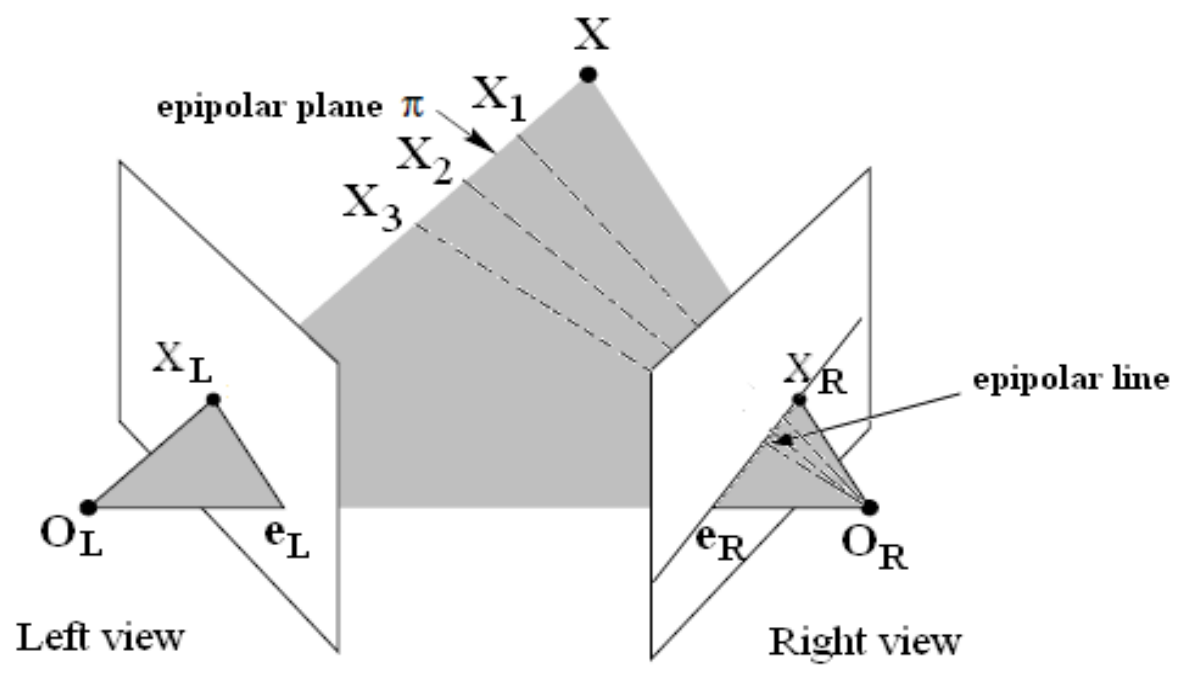

Figure 1. Example of Epipolar Geometry

\subsection{Rational Polynomial Coefficient Model}

The IKONOS Rational Polynomial Coefficient (RPC) model provides a functional relationship from the object space to the image space. The RPC functional model is of the form of a ratio of two cubic functions of the object space coordinates. Separate rational functions are used to express the relationship of the object space to the line and the object space to the sample coordinate. The line and sample RPC model are given as equation (1).

$$
\begin{gathered}
l=\frac{F_{1}(U, V, W)}{F_{2}(U, V, W)} \\
s=\frac{F_{3}(U, V, W)}{F_{4}(U, V, W)}
\end{gathered}
$$

$$
\begin{aligned}
F(U, V, W)= & a_{1}+a_{2} \cdot V+a_{3} \cdot U+a_{4} \cdot W+a_{5} \cdot V \cdot U+a_{6} \cdot V \cdot W+a_{7} \cdot U \cdot W \\
& +a_{8} \cdot V^{2}+a_{9} \cdot U^{2}+a_{10} \cdot W^{2}+a_{11} \cdot U \cdot V \cdot W+a_{12} \cdot V^{3}+a_{13} \cdot V \cdot U^{2} \\
& +a_{14} \cdot V \cdot W^{2}+a_{15} \cdot V^{2} \cdot U+a_{16} \cdot U^{3}+a_{17} \cdot U \cdot W^{2}+a_{18} \cdot V^{2} \cdot W \\
& +a_{19} \cdot U^{2} \cdot W+a_{20} \cdot W^{3}
\end{aligned}
$$

In equation (1), $F_{1}, F_{2}, F_{3}$ and $F_{4}$ are the third rational polynomial functions described by equation (2). $U, V$ and $W$ are normalized object space coordinates (latitude, longitude, height), and $l$ and $s$ are normalized image space coordinates (sample, line). [a $\left.\mathrm{a}_{1} \ldots \mathrm{a}_{20}\right]$ are rational polynomial coefficients, which are determined by fitting the physical camera model.

Suppose $(s, l)$ are the pixel coordinates of an image point, and (Lat, Lon, Height) are the geodetic coordinates of the corresponding object point, then we have the following relationships. 


$$
\begin{aligned}
& U=\frac{L a t-L a t \_O F F}{L a t \_S C A L E}, V=\frac{L o n-L o n \_O F F}{L o n \_S C A L E}, W=\frac{H e i g h t-H \_O F F}{H \_S C A L E} \\
& s=\frac{S-S_{\text {OFF }}}{S \_l C A L E}, l=\frac{L-L \_O F F}{L \_S C A L E}
\end{aligned}
$$

In equation (3), Lat_OFF, Lat_SCALE,Lon_OFF and Lon_SCALE are the normalized parameters of ground point coordinates, while $S \_O F F, S \_S C A L E, L \_O F F$ and $L \_S C A L E$ are the normalized parameters of the image point coordinates that can be provided in RPC files by the satellite vendor.

\section{Epipolar Resampling}

\subsection{PEL Determination}

An epipolar curve can be generated in the right image from an image point from the left image using inverse and forward RPC [10] by changing the ground height in Figure 2.

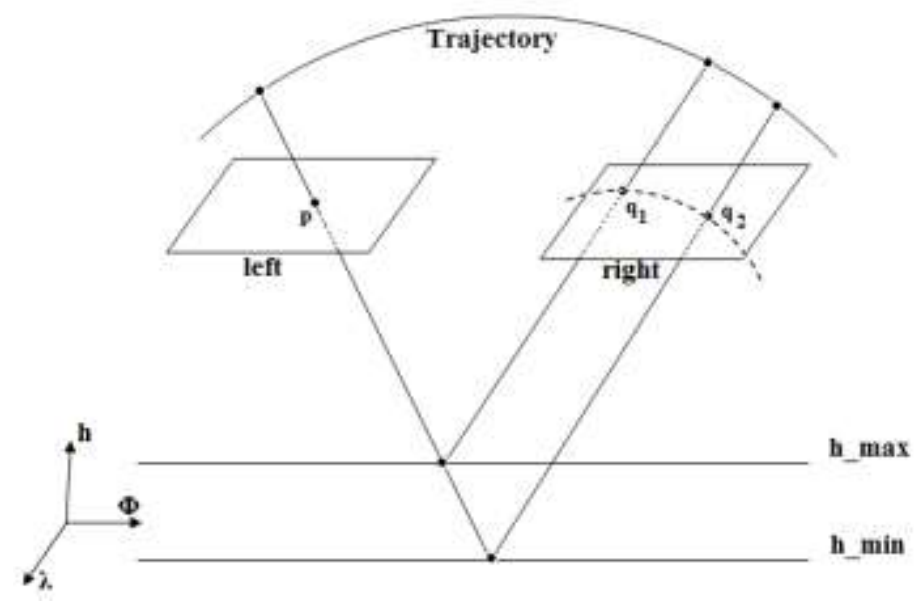

Figure 2. Epipolar Curve Generation

From an image point $\mathrm{p}$ from the left image, we calculate real world points with the ground heights of h_min and h_max using inverse projection, based on the RPC. The image space coordinates (latitude, longitude, height) are shown in Figure 2. Using forward projection, corresponding image coordinates $\mathrm{q}_{1}$ and $\mathrm{q}_{2}$ are calculated in the right image from the real world points with the ground heights of $h \_$min and $h \_m a x$. The transformation can be either a forward mapping from the image space to the target space, or an inverse mapping from the target space to the image space. The two image points $\mathrm{q}_{1}$ and $\mathrm{q}_{2}$ on the right image correspond to the left image point $\mathrm{p}$, and form an epipolar curve. Assume that the projection line from the satellite image sensor is linear. Then the epipolar line from $\mathrm{p}_{1}$ to $\mathrm{p}_{2}$ can be represented by the straight line referred to as the Pseudo Epipolar Line (PEL) [10], shown in Figure 2.

\subsection{Piecewise Linear Method}

In previous studies, epipolar lines have been approximated by a linear line. If we resample the whole image by PEL, it will not give an accurate result. This phenomenon is illustrated in Figure 3. In this context, we consider the piecewise linear implementation of PEL for accuracy improvement. The idea is illustrated in Figure 4. 
For the piecewise linear implementation, the entire image is divided into small pieces. As shown in Figure 5, we assume $\mathrm{P}_{1}$ point is selected on the first piece of the reference (left) image. Using inverse and forward RPC transformation with the height range [h min, $h$ max], we obtain two corresponding points $\left(\mathrm{q}_{1}\right.$ and $\left.\mathrm{q}_{2}\right)$ on the first piece of the target (right) image. The PEL can be generated from that two points $\left(\mathrm{q}_{1}\right.$ and $\left.\mathrm{q}_{2}\right)$ and then the point $C_{1}$ is obtained on the boundary line between the two contiguous pieces. From the point $\mathrm{C}_{1}$, we calculate the corresponding point $\mathrm{p}_{2}$ on the left image using inverse and forward RPC transformation, respectively. In this case we use h_max as the height. Then we obtain the image point $\mathrm{C}_{2}$ at the second piece on the right image by applying inverse and forward transformation with the height of $h \_m i n$. The two points $C_{1}$ and $C_{2}$ construct another PEL in the second piece. Next, the above steps are repeated to determine all the piecewise continuous PELs in the entire image.

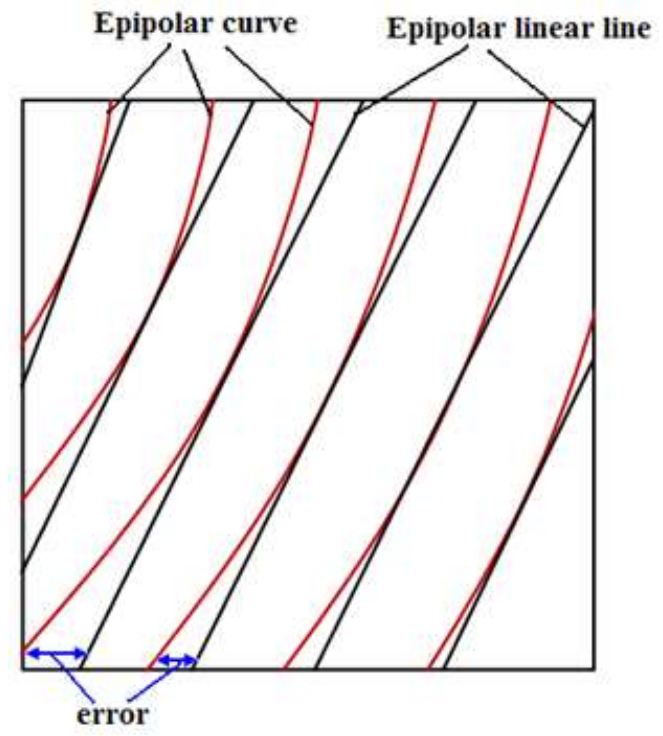

Figure 3. Epipolar Curves on the Entire Image

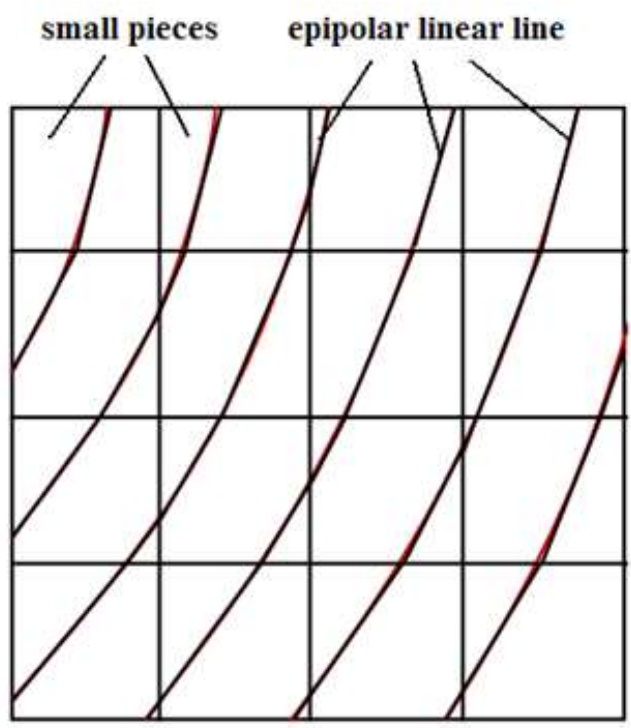

Figure 4. The Formation of Epipolar Lines on Small Pieces 


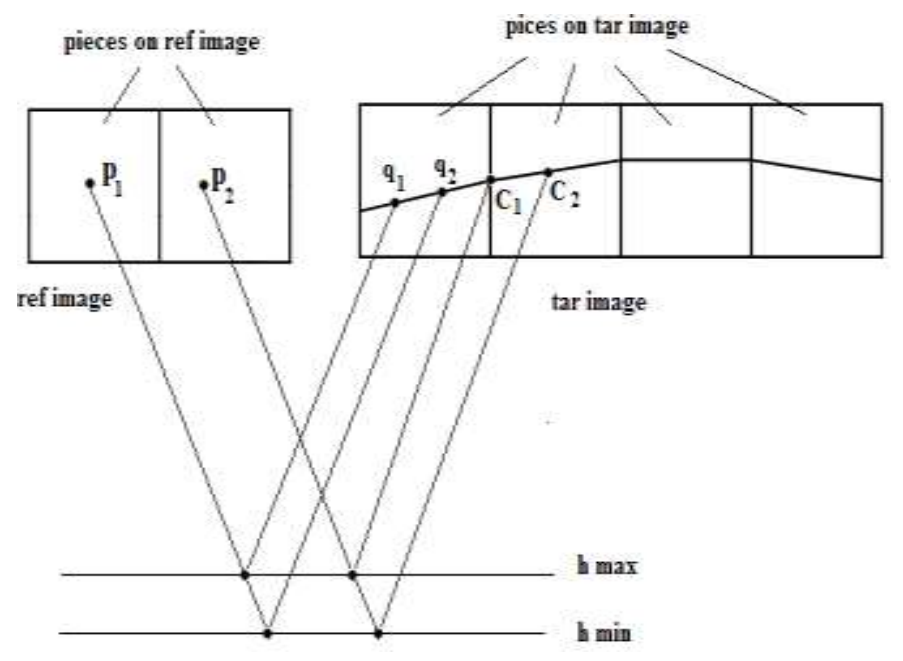

Figure 5. Piecewise Linear Method

\section{Experiments}

To resample each image with our piecewise linear method, we employed two stereo pairs of IKONOS images for the analysis and evaluation of the proposed method. Table 1 shows the information of the IKONOS stereo images used in the experiment. The two sets of stereo images are shown Figure 6 and Figure 7.

Table 1. Test Image Data

\begin{tabular}{|c|c|c|}
\hline $\begin{array}{c}\text { Data } \\
\text { information }\end{array}$ & \multicolumn{2}{|c|}{ IKONOS Stereo } \\
\hline Image Type & Pushbroom & Pushbroom \\
\hline Image Size & $11004 \times 11004$ & $11105 \times 11072$ \\
\hline Location & Daejeon, Korea & Boston, USA \\
\hline
\end{tabular}

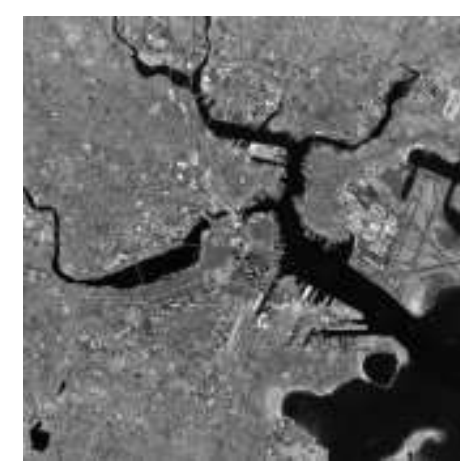

(a) Reference (Left) Image

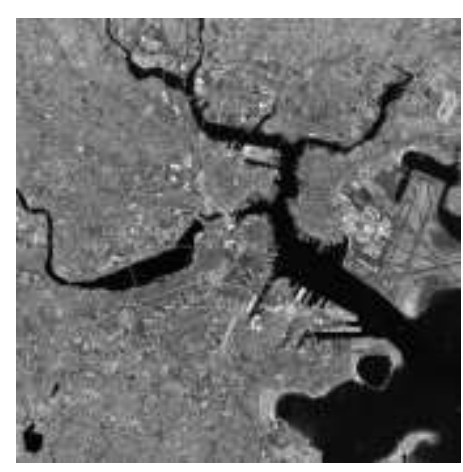

(b) Target (Right) Image

Figure 6. A Stereo Pair of Boston Images

To resample each pair of images with the piecewise linear solution, the entire image is divided into small pieces according to several levels: level 1 with one whole image, level 2 with $4 \times 4$ or 16 pieces, level 3 with $6 \times 6$ or 35 pieces and level 4 with $12 \times 12$ or 144 pieces. Figure 8, Figure 9, Figure 10 and Figure 11 are the epipolar resampled images of Boston images using levels 1, 2, 3 and 4, respectively. Similarly, Figure 12, Figure 13, Figure 14 and Figure 15 are the epipolar resampled images of Daejeon images using levels 1, 2, 3 and 4, respectively. 


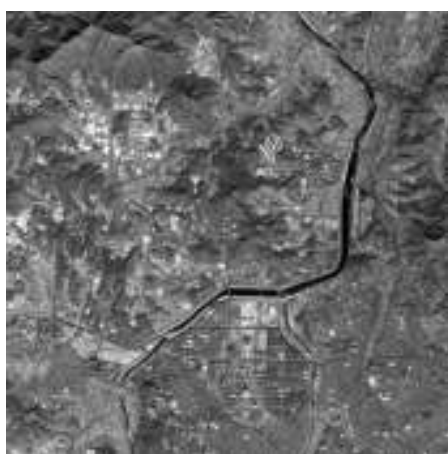

(a) Reference (Left) Image

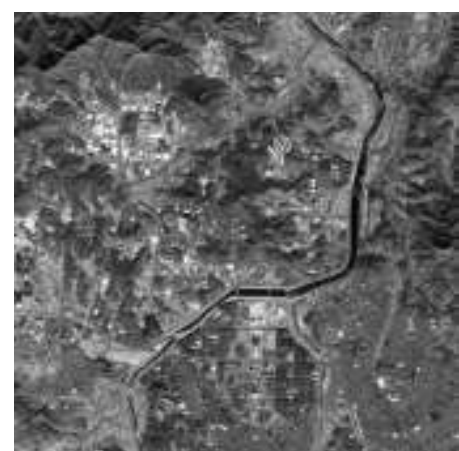

(b) Target (Right) Image

Figure 7. A Stereo Pair of Daejeon Images

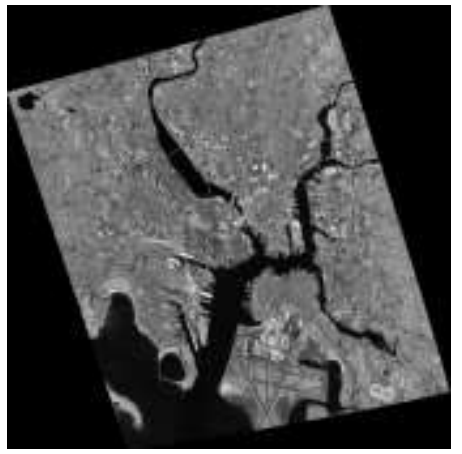

(a) Reference (Left) Image

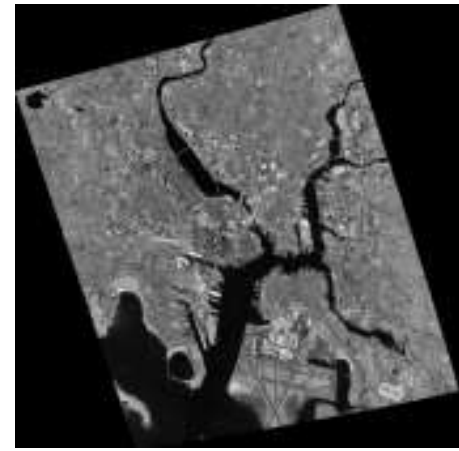

(b) Target (Right) Image

Figure 8. Boston Epipolar Images at Level 1 - Entire Image

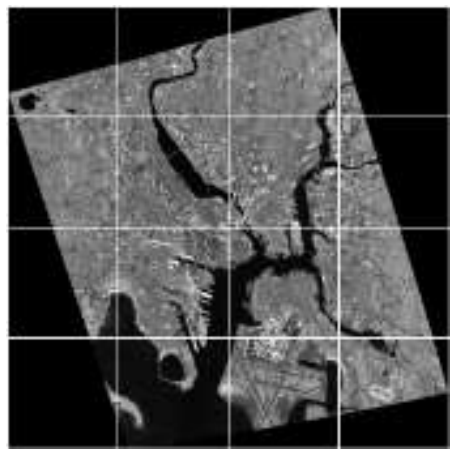

(a) Reference (Left) Image

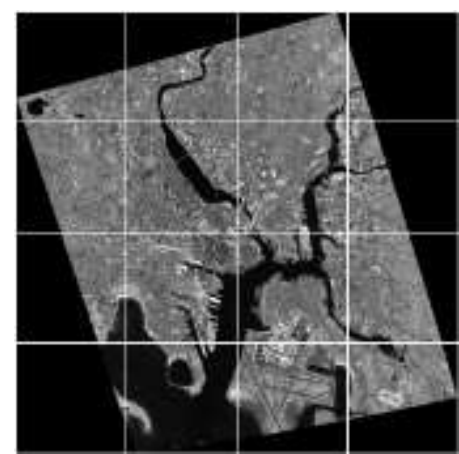

(b) Target (Right) Image

Figure 9. Boston Epipolar Images at Level 2 - 4x4 Images

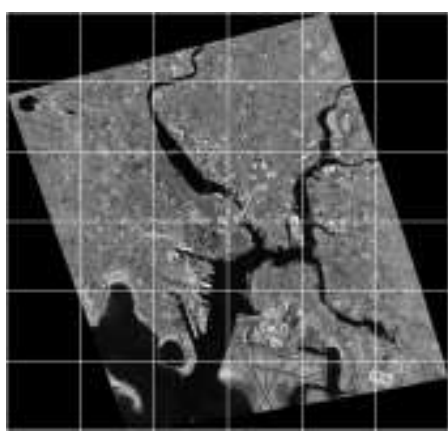

(a) Reference (Left) Image

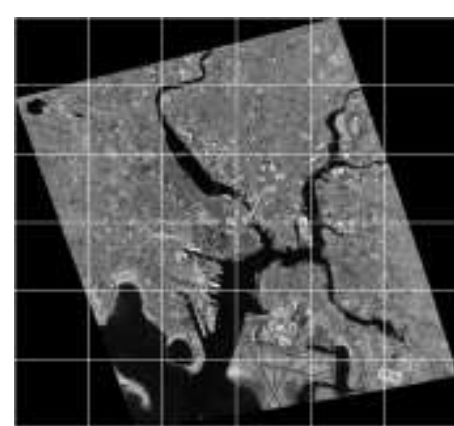

(b) Target (Right) Image

Figure 10. Boston Epipolar Images at Level 3-6x6 Images 


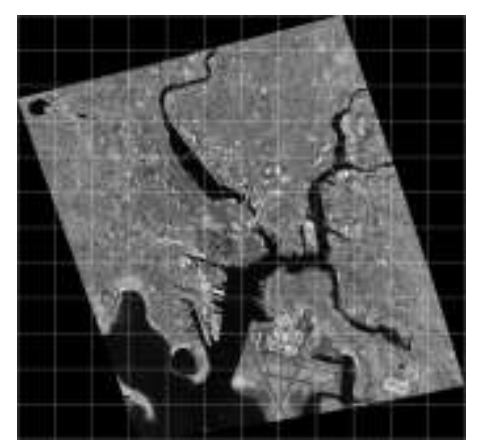

(a) Reference (Left) Image

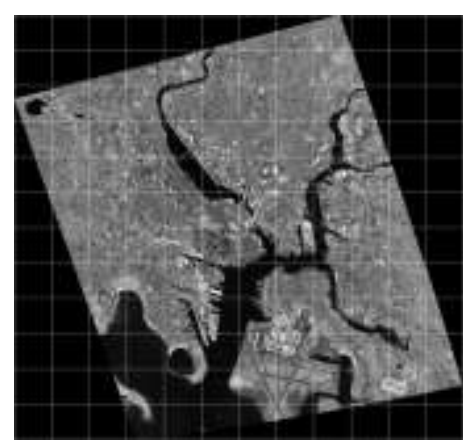

(b) Target (Right) Image

Figure 11. Boston Epipolar Images at Level 4 - 12x12 Images

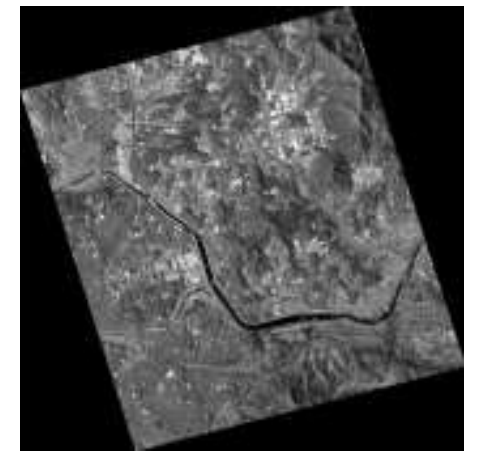

(a) Reference (Left) Image

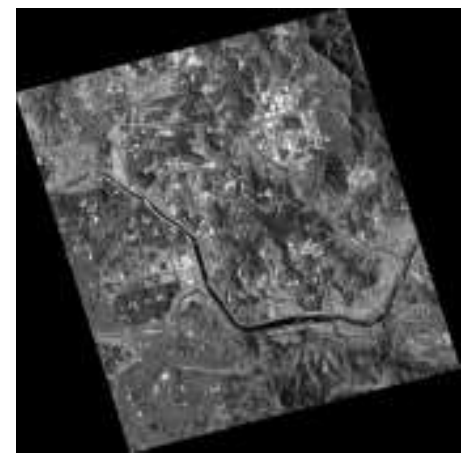

(b) Target (Right) Image

Figure 12. Daejeon Epipolar Images at Level 1 - Entire Image

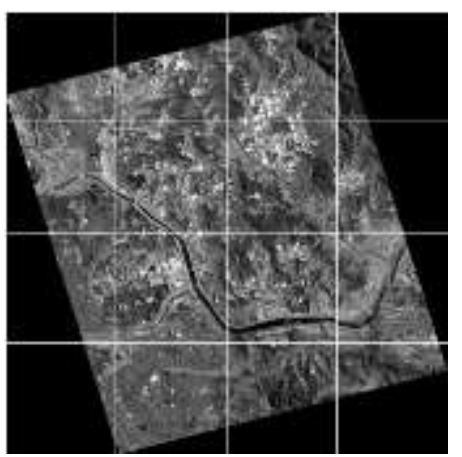

(a) Reference (Left) Image

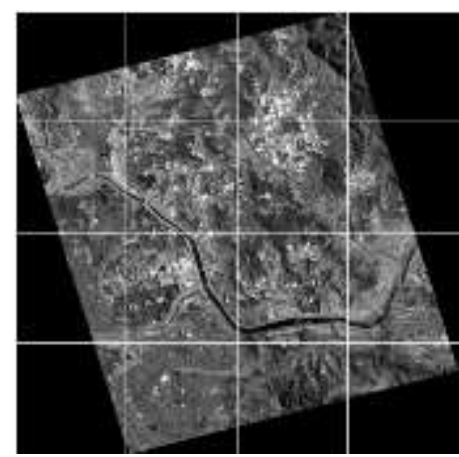

(b) Target (Right) Image

Figure 13. Daejeon Epipolar Images at Level 2 - 4x4 Images

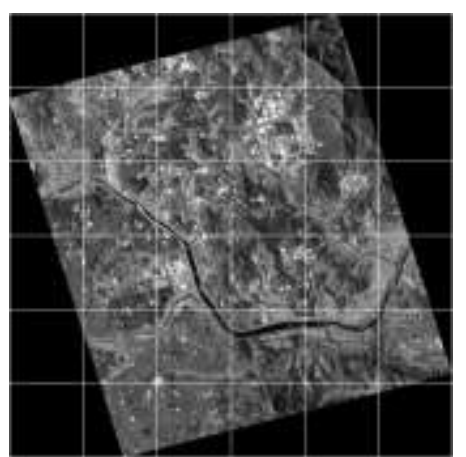

(a) Reference (Left) Image

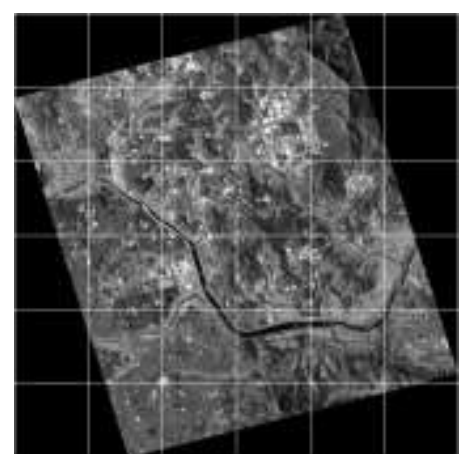

(b) Target (Right) Image

Figure 14. Daejeon Epipolar Images at Level 3-8x8 Images 


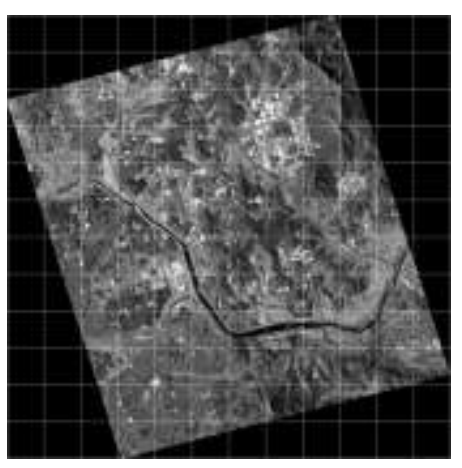

(a) Reference (Left) Image

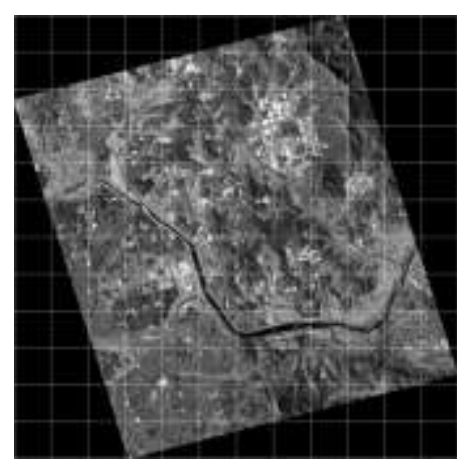

(b) Target (Right) Image

Figure 15. Daejeon Epipolar Images at Level 4 - 12x12 Images

The experiment result of the epipolar resampled image is analyzed by the calculation of building corners between two epipolar images. The corresponding points should lie on the same rows when we have ideal epipolar resampled images. Using detected building corners, we measure the horizontal deviation of corners as an epipolar resampled error. For the Boston images, we use 400 check points at level 1, 625 check points at level 2, 1046 check points at level 3 and 1384 check points at level 4. For the Daejeon images, we also use 340 check points at level 1, 363 check points at level 2, 386 check points at level 3 and 550 check points at level 4 . The result of the two pairs of epipolar images is displayed in Table 2, Table 3, Table 4 and Table 5.

Table 2. Error Points at Level 1 - Entire Image

\begin{tabular}{|c|c|c|}
\hline Location & Daejeon images & Boston images \\
\hline Image size & $11004 \times 11004$ & $11105 \times 11072$ \\
\hline Total check points & 340 & 400 \\
\hline Max error pixel & 3 pixel & 3 pixel \\
\hline Total error points & 108 & 305 \\
\hline Error percent & $31.76 \%$ & $76.85 \%$ \\
\hline Average error & 0.323 & 0.915 \\
\hline
\end{tabular}

Table 3. Error Points at Level $2-4 \times 4$ Pieces

\begin{tabular}{|c|c|c|}
\hline Location & Daejeon images & Boston images \\
\hline Image size & $2751 \times 2751$ & $2776 \times 2768$ \\
\hline Total check points & 363 & 625 \\
\hline Max error pixel & 1 pixel & 1 pixel \\
\hline Total error points & 69 & 70 \\
\hline Error percent & $19.01 \%$ & $11.2 \%$ \\
\hline Average error & 0.190 & 0.117 \\
\hline
\end{tabular}

Table 4. Error Points at Level 3-6x6 Pieces

\begin{tabular}{|c|c|c|}
\hline Location & Daejeon images & Boston images \\
\hline Image size & $1834 \times 1834$ & $1850 \times 1845$ \\
\hline Total check points & 386 & 1046 \\
\hline Max error pixel & 1 pixel & 1 pixel \\
\hline Total error points & 63 & 69 \\
\hline Error percent & $16.32 \%$ & $6.59 \%$ \\
\hline Average error & 0.163 & 0.065 \\
\hline
\end{tabular}


Table 5. Error Points at Level 4 - 12x12 Pieces

\begin{tabular}{|c|c|c|}
\hline Location & Daejeon images & Boston images \\
\hline Image size & $917 \times 917$ & $925 \times 922$ \\
\hline Total check points & 550 & 1384 \\
\hline Max error pixel & 1 pixel & 1 pixel \\
\hline Total error points & 67 & 21 \\
\hline Error percent & $12.18 \%$ & $1.52 \%$ \\
\hline Average error & 0.122 & 0.015 \\
\hline
\end{tabular}

It is noted that the accuracy of the epipolar resample is significantly improved when the entire image is divided into more pieces. In addition, the number of error points and the maximum of the error accordingly decreased. According to this analysis, our approach can be efficiently applied to the accurate and simple epipolar resampling of IKONOS satellite images. We conclude that our piecewise linear scheme is very practical and efficient for the resampling of IKONOS images.

\section{Conclusion}

The main objective of this research is to generate epipolar images obtained using a piecewise linear method. For stereo matching, epipolar images should be found as a preprocessing step to achieve reduced computation time as well as the reduction of matching ambiguities. PEL is a very practical way of epipolar resampling of pushbroom satellite images. However, this PEL approach is not a good way for a high resolution image if we resample the entire image. This is due to the fact that PEL is only an approximation of the real epipolar curve. In this context, we proposed the piecewise implementation of PEL in this paper. The experiment results showed that highly accurate resampling was achieved when the entire image was divided into multiple small pieces because our scheme is based on the conjugate points near the approximated PEL. With this accuracy, we believe that the proposed method can be efficiently utilized in the epipolar image generation of pushbroom satellite imagery.

\section{Acknowledgement}

This paper is a revised and expanded version of a paper entitled "Piecewise Linear Epipolar Resampling of IKONOS Satellite Images" presented at the 1st International Conference on Convergent Research Theory and Technology in Jeju, Korea on August 20, 2017. This work was supported by 2016 Research Fund of Myongji University.

\section{References}

[1] G. Feng, L. Ma, X. Tan, H. Xue and K. Guan, "Visual Location Recognition Based on Coarse-to-Fine Image Retrieval and Epipolar Geometry Constraint for Urban Environment", International Journal of Signal Processing, Image Processing and Pattern Recognition, vol. 9, no. 11, (2016), pp. 25-36.

[2] J. Grodecki and G, Dial, "IKONOS Geometry Accuracy", Proc. of Joint International Workshop on High Resolution Mapping from Space, Hannover, (2001), pp. 77-86.

[3] H. Pan, G. Zhang and T. Chen, "A General Method of Generating Satellite Epipolar Images Based on RPC Model”, Proc. IGARSS, (2011).

[4] T. Kim, "A Study on the Epipolarity of Linear Pushbroom Images", Photogrammetric Engineering and Remote Sensing, vol. 66, no. 8, (2000), pp. 961-966.

[5] R. Gupta and R. I. Hartley, "Linear Pushbroom Cameras", IEEE Trans. Pattern Analysis and Machine Intelligence, vol. 19, no. 9, (1997), pp. 963-975.

[6] T. Ono, "Epipolar Resampling of High Resolution Satellite Imagery", Int. Achieves Photogramm. Remote Sens. Spat. Inf. Sci., (1999).

[7] M. Morgan, K. Kim, S. Jeong and A. Habib, "Parallel Projection Modelling for Linear Array Scenes", $20^{\text {th }}$ Congress of ISPRS, (2004), pp. 12-23. 
[8] M. Morgan, K. Kim, S. Jeong and H. Ayman, "Indirect Epipolar Resampling of Scenes Using Parallel Projection Modeling of Linear Array Scanners", Int. Achieves Photogramm. Remote Sens. Spat. Inf. Sci., vol. 35, (2004), pp. 52-57.

[9] M. Wang, F. Hu and J. Li, "Epipolar Resampling of Linear Pushbroom Satellite Imagery by New Epipolarity Model”, ISPRS journal of Photogrammetry and Remote Sensing, vol. 66, no. 3, (2011), pp. 347-355.

[10] D. Woo, "Terrain Reconstruction for Site Modeling", The second year report, Agency for defense development, (2003).

[11] H. Shon, C. Park and H. Chang, "Rational Function Model-Based Image Matching for Digital Elevation Models", Photogrammetric Record, vol. 20, (2005), pp. 366-383.

[12] J. Oh, W. Lee, C. K. Toth and C. Lee "A Piecewise Approach to Epipolar Resampling of Pushbroom Satellite Images Based on RPC", Photogrammetric Engineering \& Remote Sensing, vol. 76, no. 12, (2010), pp. 1353-1363.

[13] J. Koh and H. Yang, "Unified Piecewise Epipolar Resampling Method for Pushbroom Satellite Images", EURASIP Journal on Image and Video Processing, vol. 2016:11, (2016), pp. 1-14.

\section{Authors}

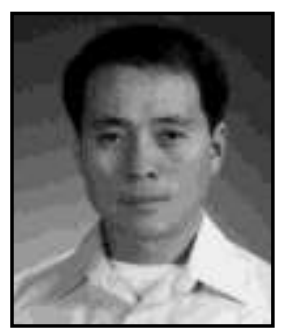

Dong-Min Woo, he got BS and MS in Electronics Engineering at Yonsei University, Seoul, Korea, respectively in 1980 and 1982. In 1987, he earned Ph.D. in Electrical Engineering and Applied Physics at Case Western Reserve University, located in Cleveland, Ohio, USA. His research interests include computer vision, computational intelligence and remote sensing.

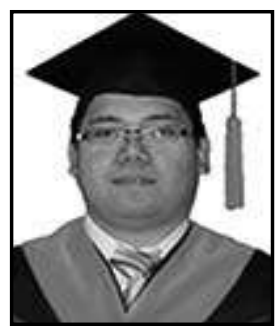

Thanh-Danh Pham, he got BS at Woosong University in 2008, and received MS at Myongji University in 2012. His research interests include computer vision, computational intelligence and multimedia library. 
International Journal of Grid and Distributed Computing

Vol. 11, No. 5 (2018) 